\title{
Ansiedad y Depresión en niños con sobrepeso y obesidad: Resultados de un Campo de Verano
}

\section{Anxiety and Depression in children with overweight and obesity: Results of a Summer's field}

\author{
Edith Gerardina Pompa Guajardo, Mónica Teresa González Ramírez¹ \& \\ Francisco Torres Guerrero \\ Facultad de Psicología, Universidad Autónoma de Nuevo León \\ (Recepción: Mayo 2010 - Aceptación: Octubre 2010)
}

\begin{abstract}
Resumen
La obesidad y el sobrepeso infantil se han incrementado en los últimos años a nivel mundial, por lo cual es importante promover un estilo de vida saludable mediante programas preventivos. El objetivo del presente artículo es evaluar la eficacia de un programa multidisciplinario que fue diseñado para la disminución del IMC, ansiedad y depresión, en niños con sobrepeso y obesidad. El estudio inicia en un campamento de verano, de 5 días de duración y se continúa con sesiones quincenales en grupo. Se presentan resultados de la evaluación inicial haciendo una comparativa con una evaluación a los seis meses de seguimiento. De acuerdo a los resultados obtenidos en el presente estudio se observa una disminución significativa en las tres variables del estudio, lo que refleja resultados satisfactorios del campo de verano y la intervención. Palabras claves: sobrepeso, obesidad infantil, programa multidisciplinario, ansiedad, depresión, campo de verano.
\end{abstract}

\begin{abstract}
Obesity and being overweight in childhood have increased in recent years at world-wide levels. Thus, it is important to promote a healthier life style by means of preventive programs. The aim of this study was to evaluate the effectiveness of the multidisciplinary program in the reduction of Body Mass Index (BMI), anxiety and depression in children with overweight and obesity problems. Our research took place in a 5-days summer camp and it continued with biweekly sessions. We show results of our initial evaluation and compare them with an evaluation performed six months following the initial evaluation. According to the results obtained in the present study a significant reduction in the three studied variables was observed.

Key words: overweight, childhood, obesity, multidisciplinary program, anxiety, depression, summer camp.
\end{abstract}

\section{Introducción}

El sobrepeso y obesidad son problemas de salud de alta prevalencia, en los últimos años la obesidad ha ido en aumento desplazando a la desnutrición y en algunas partes del mundo se ha convertido en el principal problema nutricional, al grado de considerarse una pandemia que afecta a más de mil millones de personas (American Obesity Association, 2000).

1 Correspondencia dirigida a: Mónica Teresa González Ramírez. Dr. Carlos Canseco 110. Col. Mitras Centro. C. P. 64460. Tel. (52-81) 83338233. Monterrey, Nuevo León, México. E-mail: monygzz77@yahoo.com, monica.gonzalezrz@uanl. edu.mx. 
La Organización Mundial de la Salud en 1998 declaró a la obesidad como una epidemia global que incluye niños y adultos, por lo que la prevención es fundamental ya que si se comienza desde la infancia podrá promover un estilo de vida más saludable (Edmunds, Waters \& Elliot, 2001).

Un importante incremento en la prevalencia de obesidad infantil la ha vuelto un problema de salud publica en donde lo considerable son las consecuencias a corto plazo como alteraciones del sueño, asma, pobre imagen de sí mismo y ansiedad entre otras. A largo plazo produce un aumento paralelo de enfermedades crónicas asociadas como hipertensión arterial, dislipidemias, diabetes mellitus tipo 2, ateroesclerosis, problemas ortopédicos como pie plano, escoliosis, problemas psicosociales, derivados de la falta de aceptación social y baja autoestima, observándose lo anterior cada vez en edades más tempranas en la infancia (Silvestri y Stavile, 2005).

La Encuesta Nacional de Salud y Nutrición (2006) en México indica que el 26\% de los niños padecen obesidad o sobrepeso. Esto resulta alarmante ya que si estos niños llegan a la adolescencia con obesidad muy probablemente lo seguirán en la edad adulta con el riesgo sobreañadido de una mayor morbimortalidad.

Aun no se conocen los mecanismos fisiopatológicos causantes de la obesidad y aunque existen factores genéticos, el incremento de la obesidad como trastorno nutricional se ve claramente asociado a un aumento en la ingesta calórica y una marcada disminución en la actividad física, es decir un cambio en el estilo de vida (Vela et al., 2009).

Es importante abordar el aspecto psicológico, ya que muchos trastornos del apetito encubren disfunciones familiares o problemas de autoestima y sociabilidad, los que pueden influir en las conductas con respecto a ingesta y actividad física, este efecto de baja autoestima característico en niños con obesidad es señalado por Paxton (2005) quien indica que la obesidad infantil tiene un riesgo significativo para el desarrollo de problemas de salud, pero también el aspecto psicológico y social tiene consecuencias graves en donde la baja autoestima se asociada con depresión.

Estudios como el de Jonides, Buschbacher \& Barlow, (2002) han examinado la relación de la obesidad con aspectos psicológicos como baja autoestima depresión y altos niveles de síntomas emocionales y enfatizan la importancia de estudiar estas variables psicológicas en personas con obesidad. Así, se ha encontrado que la pobre imagen corporal es uno de los factores psicológicos más frecuentes en los niños obesos y está en relación con una baja autoestima, depresión, y bajo funcionamiento psicosocial (Parysow, 2005).

En cuanto a los tratamientos Durá \& Sánchez- Valverde (2005) mencionan que el tratamiento para la obesidad infantil debe ser multidisciplinar y abarcar cambios de estilo de vida, tanto en la escuela como en la casa y que la parte más importante es la prevención que evitará el aumento de prevalencia de este problema. Asimismo, debido a que el ser humano es un ser bio-psico-social se deben tomar en cuenta todos los aspectos involucrados, genéticos, constitucionales, ambientales, sociales considerando la cronicidad y complejidad de este problema de salud. De tal modo un programa de intervención no solo debe ser multidisciplinario, sino que también debe adaptarse a las condiciones de la comunidad en que se implementa. Las cuales en muchas ocasiones, distan de ser las circunstancias ideales para lograr los resultados deseados.

Con base en lo anterior, el objetivo del presente artículo es evaluar la eficacia del programa multidisciplinario en la disminución del IMC, ansiedad y depresión, a los seis meses de su inicio.

\section{Método}

\section{Participantes}

Se inició el campo de verano con 20 niños inscritos, de los cuales solo terminaron 14. Se obtuvo el consentimiento informado de los padres o tutores de todos los niños, se valoró el IMC mediante 
una balanza de precisión Tanita Inner Scan BC 418, y de acuerdo a los estándares de Cole et al., (2000) se elaboraron puntos de corte para el IMC para niños y adolescentes.

\section{Procedimiento}

El reclutamiento de sujetos se realizó mediante la promoción en diferentes medios de comunicación. Se obtuvo el consentimiento informado de los padres o tutores de los niños incluidos en el estudio.

Se valoró el peso por medio de una balanza de precisión (Tanita Inner Scan BC 418), el perímetro abdominal mediante una cinta métrica y la talla por medio de un estadiómetro, estos datos fueron evaluados por una misma persona y con base en ellos se obtuvo el Índice de Masa Corporal (IMC).

Se les realizaban cuatro intervenciones a los niños, pediátrica para evaluar el estado fisco del niño que eran al inicio y al final; nutricional cada quince días; medicina del deporte donde se realizó una prueba de esfuerzo al inicio y sesiones cada quince días para la promoción de una actividad física y la intervención psicológica que fue de la siguiente manera: se les veía cada quince días con sesiones psicoeducativas que duraban una hora y media en forma grupal, en las cuales los niños intercambiaban temas o situaciones que les hubieran pasado durante ese periodo en donde además escribían en un diario cómo se habían sentido durante todo ese tiempo; esta actividad permitió el intercambio de experiencias de cada uno de los participantes, ya que al momento de platicar con los demás aprendían y se dio un proceso de identificación, en el cual los niños se daban cuenta de que otros sentían lo mismo y aprendían de la forma en que resolvían las situaciones.

La intervención con los padres era una vez al mes en donde al inicio se les permitía que expresaran que experiencia habían tenido con respecto a sus hijos (control de la alimentación) y al final se les explicaba en forma amplia los problemas físicos y psicológicos que los niños con obesidad podrían desarrollar a corto, mediano y largo plazo. Se enfatizaba la importancia de establecer buena comunicación con sus hijos para poder inculcarles la importancia de una alimentación saludable y la realización de algún tipo de ejercicio físico.

Además del IMC, se evaluó de forma basal mediante dos tests psicológicos, el cuestionario de Depresión para Niños de M. Lang y M. Tisher (1983) y la escala de Ansiedad manifiesta en Niños de Cecil R. Reynolds y Bert O. Richmond (1997), después de seis meses de sesiones grupales cada quince días, se volvieron a aplicar los mismos instrumentos para comparar resultados.

\section{Instrumentos}

El CMAS-R de Reynolds y Richmond (1997) se diseñó para aplicarse en forma individual o grupal en niños entre 6 hasta 18 años. Es un instrumento de auto informe que consta de 37 reactivos diseñado para valorar el nivel y la naturaleza de la ansiedad en niños y adolescentes. La suma de las respuestas positivas, nos determina la puntuación de ansiedad total. Reynolds y Richmond reportan que la escala cuenta con consistencia interna adecuada, con un valor de .83 estimado con la fórmula Kuder-Richardson para ítems dicotómicos. Asimismo, en el manual de la escala se reportan numerosos estudios con evidencia de consistencia interna adecuada. Finalmente, se reporta una gran cantidad de evidencia respecto a la validez de constructo, concurrente y divergente. Es así que la escala cuenta con adecuadas propiedades psicométricas.

La Escala de Depresión para niños CDS también es un cuestionario autoaplicable desarrollado por Lang y Tisher en 1983, consta de 66 ítems. En cuanto a sus propiedades psicométricas el análisis de validez de contendido se utilizó en la edición anterior del cuestionario, para el criterio y discriminación de una muestra de niños con depresión y el juicio de siete psiquiatras infantiles, para la validez concurrente se comparó con el Cuestionario de Personalidad CPQ de Catell y capacidad discriminativa con un criterio de infelicidad, para la validez de constructo se llevó a cabo mediante un análisis de diferencias entre los grupos experimental, control y clínico. En la versión española su índice de confiabilidad fue mediante la formulación de Kuder-Richarsdson (K-R20) obteniendo un valor de .91 , en una muestra de 730 niños de ocho a catorce años. 


\section{Resultados}

El estudio se realizó con 14 niños de los cuales 6 eran niñas (42.9\%) y 8 niños (57.1\%), con una media de edad de 10.14, una mediana de 10 y una desviación estándar de 1.231 , con respecto al grado escolar la media fue de 4.50 una mediana de 4.50 y 1.557 de desviación estándar (Tabla 1).

Tabla 1. Descripción de la muestra

\begin{tabular}{lccc}
\hline & & Grupo $2008(\mathrm{n}=14)$ & \\
& Media & Mediana & DE \\
\hline Edad & 10.14 & 10.00 & 1.231 \\
Grado escolar & 4.50 & 4.50 & 1.567 \\
Sexo & & Varones $=6$ & \\
& & Mujeres $=8$ & \\
\hline
\end{tabular}

La descripción de los puntajes obtenidos en ansiedad, depresión e IMC en las diferentes evaluaciones se muestran en la tabla 2, puede observarse una disminución en las tres variables a los seis meses en comparación al puntaje inicial.

Tabla 2. Estadísticos descriptivos para las variables de estudio y prueba de normalidad

\begin{tabular}{|c|c|c|c|c|c|}
\hline & \multicolumn{5}{|c|}{ Grupo $2008(n=14)$} \\
\hline & Media & Mediana & $\mathrm{DE}$ & $\mathrm{K}-\mathrm{S}_{\mathrm{z}}$ & $\mathrm{p}$ \\
\hline $\begin{array}{l}\text { IMC } \\
\text { inicial }\end{array}$ & 28.10 & 29.20 & 4.64 & 0.16 & .200 \\
\hline a los 6 meses & 27.32 & 28.05 & 4.70 & 0.13 & .200 \\
\hline \multicolumn{6}{|l|}{ Ansiedad } \\
\hline inicial & 11.93 & 12.00 & 4.46 & 0.10 & .200 \\
\hline a los 6 meses & 7.43 & 8.00 & 3.92 & 0.17 & .200 \\
\hline \multicolumn{6}{|l|}{ Depresión } \\
\hline inicial & 126.64 & 120.00 & 35.18 & 0.17 & .200 \\
\hline a los 6 meses & 106.79 & 106.50 & 26.31 & 0.17 & .200 \\
\hline
\end{tabular}

Se evaluó mediante la prueba de Kolmogorov- Smirnov si las variables se ajustaban o no a una distribución normal a pesar de que la muestra es pequeña se utilizaron pruebas paramétricas debido a que hubo un ajuste a la distribución normal en las variabas de estudio (Tabla 2).

La prueba t de Student para muestras relacionadas proporciona evidencia de que la disminución en los puntajes de IMC ansiedad y depresión fue significativa estos resultados se muestran en la tabla 3. 
Tabla 3. Comparación de medias al inicio y a los 6 meses de la intervención

\begin{tabular}{lcc}
\hline $\begin{array}{l}\text { Prueba } \mathrm{t} \text { de Student } \\
\text { de muestras relacionadas }\end{array}$ & $\mathrm{t}$ & $\mathrm{p}$ \\
\hline IMC inicial / 6 meses & 3.404 & .005 \\
Ansiedad inicial / 6 meses & 3.859 & .002 \\
Depresión inicial / 6 meses & 3.507 & .004 \\
\hline
\end{tabular}

Al observar las medidas de tendencia central de IMC (Tabla 1), puede notarse que la disminución, a pesar de ser significativa (Tabla 3), es leve. Es por esto que se realizó una comparación sujeto por sujeto para identificar los cambios a los seis meses. En la figura 1 se muestra dicha comparación. Si bien, la disminución es leve o en algunos casos no hay cambio, ninguno de los sujetos tuvo un incremento en IMC.

Figura 1 Índice de Masa Corporal al inicio y a los seis meses

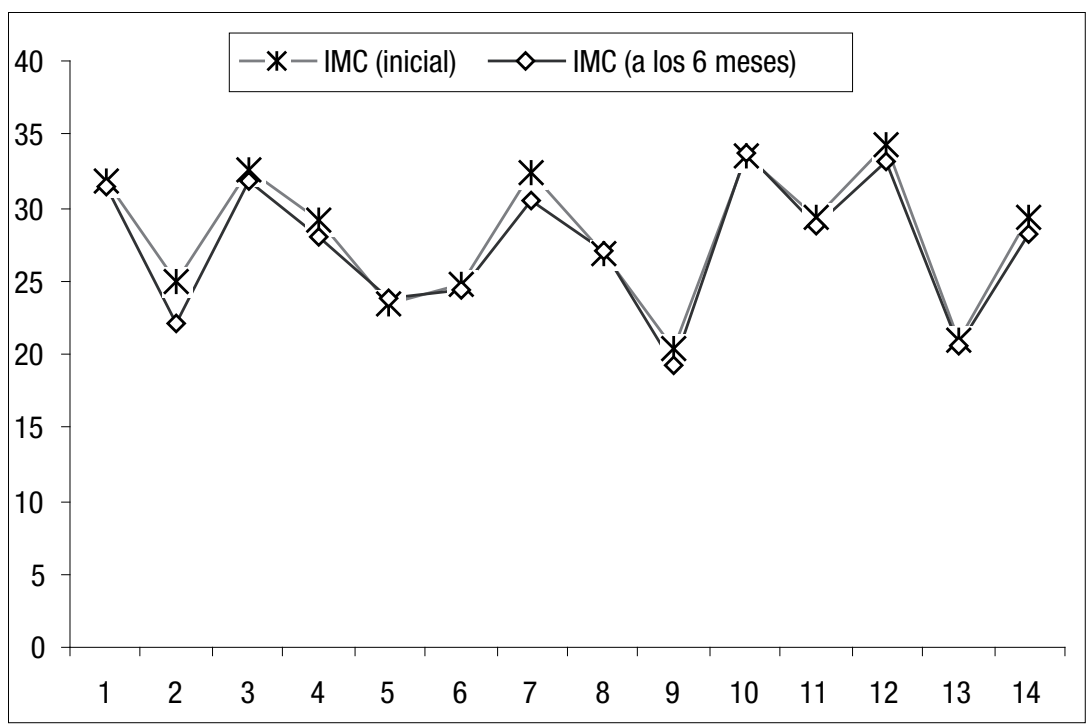

\section{Discusión}

De acuerdo a los resultados obtenidos en el presente estudio se observa una disminución en IMC, ansiedad y depresión, consideramos que la educación nutricional y la importancia de promover una actividad física, así como el espacio psicológico que los niños tuvieron en donde ellos podían hablar sobre como vivían su obesidad y su relación con la comida; son los puntos a considerar como relevantes en estos resultados.

Dada la evolución de la obesidad infantil en los últimos años, resulta importante trabajar en el área psicológica para poder comprender los distintos aspectos que se ponen en juego en esta enfermedad a la hora de tratarla, sobre todo considerando la cronicidad y complejidad de esta proble- 
mática (Sivestri y Stavile, 2005). Es así que la evaluación y tratamiento de la ansiedad y depresión asociadas a la obesidad son un aspecto central en un programa multidisciplinario.

Un aspecto que se incluyó en el programa evaluado, fue la familia, sin embargo, en algunos casos no se logró un apoyo total de la familia de los participantes. Es importante abordar el sobrepeso y la obesidad infantil para promover un estilo de vida saludable involucrando a la familia como el principal factor ambiental, junto con la escuela desplegando estrategias de prevención (Edmunds, Waters \& Elliott, 2001). La importancia que juegan los padres en el tratamiento es central, ya que son ellos los que introducen al niño los hábitos de alimentación, por su parte, el medio escolar se involucra en dos aspectos, por un lado el promover la actividad física y por otro, el tipo de alimentos que se venden en las cooperativas escolares. Ambos, familia y escuela, deben considerarse en el desarrollo de programas que promuevan la adquisición de hábitos alimentarios saludables y estilos de vida que se irán consolidando a lo largo de la infancia.

En el tratamiento de la obesidad infantil según Yeste et al., (2008) la prevención es prioritaria ya que una modificación en los hábitos de alimentación, el estimular la actividad física y un soporte emocional son los pilares fundamentales, para lograr con un tratamiento integral su eficacia a corto y mediano plazo. En esta prevención, así como en el tratamiento, el involucramiento de diferentes profesionales como pediatras, endocrinólogos, nutriólogos y psicólogos con un abordaje de tratamiento de trabajo grupal en educación para la salud, con la aplicación de estrategias cognitivo- conductuales y un enfoque familiar sistémico es la recomendación de autores como Martínez (2005).

Por su parte Gussinyé (2005) aplicó un programa de tratamiento integral para la obesidad infantil denominado "Niños en Movimiento" con el objetivo de trabajar una dieta mediterránea, para la disminución del índice de masa corporal, y la mejoría de los rasgos de depresión, ansiedad y una mayor satisfacción corporal mediante técnicas cognitivo conductuales. En dicha intervención, al igual que en la nuestra, se muestran resultados satisfactorios y se hace énfasis en la evaluación y tratamiento de la depresión y ansiedad.

Todo esto nos permite concluir la importancia de una atención integral (Gussinyé, 2005; Jonides, et al., 2002; Martínez, 2005; Vela et al., 2009) en donde cada uno de los diferentes profesionales trabajen en forma conjunta para la atención y prevención de un problema de salud como lo es el sobrepeso y la obesidad, cada vez más frecuente en nuestra comunidad, y que este tipo de trabajo sirva como concientización y prevención de problemas de salud física y psicológica en los niños.

\section{Referencias}

American Obesity Association. (2000). Cecil Textbook of Medicine (21 ed.) New England : W.B. Saunders Company.

Cole, T.; Bellizi, M., \& Flegal, K. (2000). Establishing a standard definition for child overweight and obesity worldwide international survey. British Medical Journal, 320, 1240-1243.

Durá, T. \& Sánchez-Valverde, F. (2005). Obesidad infantil: ¿un problema de educación individual, familiar o social? Nutrición Infantil Pediátrica, 63, 204-207.

Edmunds, L.; Waters, E. \& Elliot, E. (2001). Manejo de la obesidad infantil basado en la evidencia. British Medical Journal 323, 916- 919.

Gately, P.; Cook, C.; Barth, J.; Bewick, B.; Radley, D. \& Hill, A. (2005). Children Residential Weight- Loss Programs Can Work: A Prospective Cohort Study of Short- term Outcomes for Overweight and Obese Children. Pediatrics. 116 (1), 73-77.

Gussinyé, S. (2005). Aplicación del programa de tratamiento integral para la obesidad infantil "Niños en Movimiento. España. Tesis Inédita Universidad Autónoma de Barcelona. 
Jonides, L.; Buschbacher, V. \& Barlow, S. (2002). Management of Child and Adolescent Obesity: Psychological, Emotional, and Behavioral Assessment. Pediatrics, 110, 215- 221.

Martínez, A. (2005). Prevención integral de la obesidad infantil; el Plan Andaluz. Revista Pediatría de Atención Primaria, 7 (1), 21- 34.

Olaiz, G.; Rivera, J.; Shamah, T.; Rojas, R.; Villalpando, S.; Hernández, M. \& Sepúlveda, J. (2006). Encuesta Nacional de Salud y Nutrición 2006. Cuernavaca, México: Instituto Nacional de Salud Pública.

Parysow, R. (2005). Aspectos psicológicos en Obesidad. Un intento de interpretación. Tesinas de Belgrano. Universidad de Belgrano.

Paxton, H. (2005). The effects of childhood obesity on self-esteem. Tesis para obtener el grado de Education Specialist School Psychology. Marshall University.

Sivestri, E. \& Stavile, A. (2005). Aspectos Psicológicos de la obesidad: Importancia de su identificación y abordaje dentro de un enfoque interdisciplinario. Tesis Inédita Universidad Autónoma de Favaloro.

Vela, A.; García, Z.; Goñi, A.; Suinaga, I.; Aguayo, A.; Rica, I. \& Martul, P. (2009). Plan de intervención en la prevención de obesidad infantil "ErosotasunaZainduz" Revista Española de Obesidad, 7 (2), 91-96.

Yeste, D.; García-Reyna, N.; Gussinyer, S.; Marhuenda, C.; Clemente, M.; Albisu, M.; Gussinyer, M. \& Carrascosa, A. (2008). Perspectivas actuales del tratamiento de la obesidad infantil. Revista Española de Obesidad 6 (3), 139-152. 
